\section{Outcome of Prehospital Patients Declining Transport After Receiving Advanced Life Support Interventions}

Delbridge TR, Garrison HG, Cox RE, Paris PM

Division of Emergency Medicine, University of Pittsburgh

School of Medicine, Pittsburgh, Pennsylvania, USA

Purpose: Medicolegal risk has been associated with prehospital patients who are not transported. Yet, definitive treatment without transport of selected prehospital patients may be an effective health care, cost-savings strategy. To identify types of patients that might be considered for such a practice, the outcomes of patients who required prehospital advanced life support (ALS) interventions, but declined transport to a hospital, were examined.

Methods: Over a five-month period, structured follow-up interviews were conducted for a prospective case series of consecutive patients $(n=76$ ) who declined transport after receiving prehospital ALS interventions from an urban, municipal, AISEMS system. ALS interventions included administration of parenteral dextrose, glucagon, naloxone, diphenhydramine, and epinephrine; nebulized albuterol; and sublingual nitroglycerin. Results: Follow-up was achieved in $74(97 \%)$ patients. Treatment by EMS was for hypoglycemia (44.6\%), wheezing $(41.9 \%)$, narcotic overdose $(8.1 \%)$, chest pain $(2.7 \%)$, and allergic reaction $(2.7 \%)$. Outcomes were assessed for the first 72 hours post-ALS intervention. There were no deaths; $63(85 \%)$ had no recurrence of symptoms. One hypoglycemic patient received glucagon from paramedics a second time, 48 hours after initial ALS intervention. Seven $(9.4 \%)$ patients presented for ED evaluation; five (four wheezing and one chest pain) were admitted. Seventy $(94.6 \%)$ patients were able to resume usual activities within 72 hours. Seventy-three $(98.6 \%)$ patients were satisfied with the paramedics' treatment. Thirty-eight (51.4\%) had previously required prehospital ALS treatment but declined transport.

Conclusions: Follow-up of patients who receive prehospital ALS interventions but decline transport is achievable and should be further evaluated for routine practice. Furthermore, the results suggest that prehospital hypoglycemic patients and select wheezing patients incur no significant health risks by declining transport. Prospective clinical trials are necessary to determine the safety and cost-effectiveness of definitively treating and not transporting selected patients in various settings.

\section{Prospective Analysis of the Impact of Transport Interval on Prehospital Advanced Airway Procedures in Severely Injured Trauma Victims}

\section{Spaite DW, Valenzuela TD, Criss EA, Meislin HW, Geare EA}

Arizona Emergency Medicine Research Center,

University of Arizona, College of Medicine, Tucson, Arizona Tucson Fire Department, Tucson, Arizona, USA

Hypothesis: The likelihood of performing prehospital advanced airway procedures (PAAP) on major trauma victims is impacted by the distance (time) from the trauma center.

Methods: Prospective evaluation of PAAP (oral/nasal intubation, cricothyrotomy) using data from EMS and hospital patient records ( 16 months) in a medium-sized urban EMS system. Forty-seven consecutive, severely injured patients (ISS $>15$, Glasgow Coma Scale [GCS] <8) brought to a Level-I trauma center by fire department paramedics. Statistical analysis was Student's t-test, ANOVA, and Chi-square with Yates' correction.

Results: A total of 33 patients were male $(70.2 \%)$ with mean age of 38 years. Thirty-one patients $(66.0 \%)$ had PAAP attempted (29 successful; 93.6\%). All other patients had BLS airway support only. Mean transport interval (TI) for PAAP+ patients was 9.1 minutes compared to 5.4 minutes for PAAP ( $p=$ $.05)$. TI did not correlate with trauma score $(p=.407)$, GCS $(p$ $=.435)$, ISS $(p=.486)$, traumatic arrest $(p=.436)$, or subsequent fatality $(p=.343)$. Comparison of patients with TI $<8$ min vs $\geq 8 \mathrm{~min}$ revealed the following:

$\begin{array}{lcccc}\text { TI } & \begin{array}{c}\text { PAAP } \\ \text { ISS }>\text { 30 }\end{array} & \begin{array}{c}\text { Mortality } \\ (\%)\end{array} & \begin{array}{c}\text { Prehospital } \\ \text { Arrest } \\ (\%)\end{array} \\ <8 \text { min } & 50.0 & 34.6 & 57.5 & 26.9 \\ \geq 8 \text { min } & 85.7 & 38.1 & 52.4 & 33.3 \\ \rho \text {-value } & .024 & .953 & .945 & .875\end{array}$

Conclusion: Among critically injured patients with markedly depressed level of consciousness, greater TI increased the likelihood of PAAP, independent of injury severity parameters in a medium-sized urban EMS system. 